\title{
EISA-MRM: Multiple Reaction Monitoring with Single Quadrupole Mass Spectrometry
}

\author{
Jingchuan Xue ${ }^{\dagger}$, Rico J.E. Derks, William Webb ${ }^{\dagger}$, Aries Aisporna ${ }^{\dagger}$, Martin Giera*•, \\ and Gary Siuzdak*广\$
}

Scripps Center for Metabolomics, The Scripps Research Institute, 10550 North Torrey Pines Road, La Jolla, California 92037, United States

-Leiden University Medical Center, Center for Proteomics and Metabolomics, Albinusdreef 2, 2333ZA Leiden, Netherlands

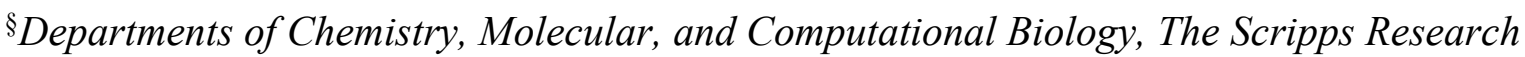
Institute, 10550 North Torrey Pines Road, La Jolla, CA 92037, United States

*Corresponding authors: m.a.giera@lumc.nl and siuzdak@scripps.edu

\section{TOC Image}

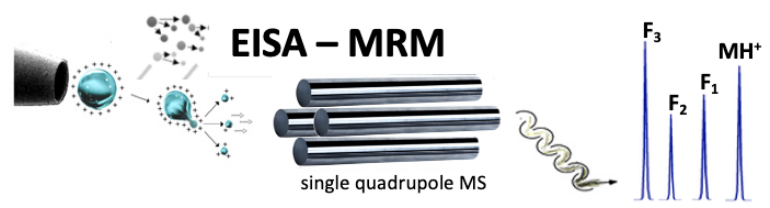

\begin{abstract}
Enhanced in-source fragmentation annotation combined with single quadrupole multiple reaction monitoring (EISA-MRM) has been designed for quantitative mass spectrometry analysis. EISA contrasts to traditional electrospray as a soft ionization technology and is proving to be advantageous since the resulting fragment ions are identical as those generated in tandem mass spectrometry. Criteria established by the European Union Commission Directive 2002/657/EC for electron ionization single quadrupole quantitative analysis was used for the EISA-MRM quantitative analyses and experiments were performed on multiple types of complex samples that included a mixture of 50 standards, as well as cell and plasma extracts. The dynamic range for quantitative analysis was comparable to QqQ-MRM analyses at up to 5 orders of magnitude and the EISA-MRM and QqQ-MRM of the cell and plasma extracts showed similar matrix effects. Amino acid and fatty acid measurements performed from certified NIST 1950 plasma with isotopically labelled standards demonstrated EISA-MRM accuracy in the range of 91-110\% for the amino acids, $76-129 \%$ for the fatty acids, and precision with a CV $<10 \%$. In order to enhance specificity and sensitivity, a newly developed Correlated SIM Chromatogram (CSC) algorithm was designed to facilitate MRM quality analyses. The EISA-MRM quantitative analysis with CSC informatics enables both precursor and in-source fragment ions to be correlated within a single quadrupole mass spectrometer.
\end{abstract}




\section{INTRODUCTION}

Triple quadrupole multiple reaction monitoring (QqQ-MRM) mass spectrometry has existed since the $1970 \mathrm{~s}^{1,2}$, and with the advent of soft atmospheric pressure ionization in the late 1980s QqQ-MRM became the primary approach for targeted small molecule analysis ${ }^{3}$. In the last three decades QqQ-MRM has dominated the mass spectrometry landscape with its ultrahigh selectivity, sensitivity, and broad dynamic range, especially in the quantification of small molecules and peptides ${ }^{4-7}$. MRM exploits the unique capability of triple quadrupole (QqQ) mass spectrometers to act as a double mass filter, facilitating the analysis of analytes from complex matrices. In MRM mode, a predetermined precursor ion is selected/isolated with the first quadrupole, then fragmented in the collision cell (second quadrupole) with a neutral gas (e.g. nitrogen $)^{8}$. Thus, only precursor derived fragment ions pass to the third quadrupole and reach the detector. The precursor-product ion pairs are referred to as 'transitions' and over a hundred transitions can be recorded in (scheduled) MRM analyses, enabling the simultaneous "targeted" tandem mass spectrometry (MS/MS) analysis of multiple analytes ${ }^{9}$. QqQ-MRM is considered the gold standard in the quantitative analysis of small molecules ${ }^{10}$ however, these instruments are typically expensive and suffer from inherent sensitivity losses in the collision chamber. Less expensive instrumentation, offering quantitative analysis for broader implementation would provide a technically advantageous alternative option.

Two separate technologies: 1) soft atmospheric pressure ionization with enhanced insource fragmentation/annotation (EISA) and 2) an MRM approach that is collectively called EISA-MRM, have been combined to provide a quantitative analysis technology in a single quadrupole MS system in which QqQ-MRM is not available (Figure 1). EISA is a recently introduced high energy electrospray ionization in-source fragmentation/annotation approach that promotes the generation of both molecular ions and their respective fragments at high abundance ${ }^{11,12}$. EISA contrasts to traditional electrospray as a soft ionization technology and is proving to be advantageous since the resulting fragment ions are typically the same as those generated in tandem mass spectrometry experiments ${ }^{11,12}$. This similarity enables the simultaneous monitoring of both precursor ion and its fragments possible in a single quadrupole MS system.

The other component of this technology, MRM using a single quadrupole mass analyzer, is a variation on an approach that has been widely used for high sensitivity detection and 
quantitative analysis of small molecules especially with gas chromatography mass spectrometry $(\mathrm{GC} / \mathrm{MS})^{13}$. However, the combination of EISA and MRM in a single quadrupole LC/MS instrument offers a unique opportunity to provide enhanced selectivity, specificity, and sensitivity for the quantitative analyses of a broader range of compounds (over traditional GC/MS). In addition, as compared with QqQ-MRM, EISA-MRM analysis performed with a single quadrupole instrumentation is available at $\sim 30 \%$ the cost, and have enhanced signal since the single quadrupole instruments do not have a collision cell and therefore do not experience the associated collision cell ion losses.

In this work, we demonstrate that EISA-MRM performed with a single quadrupole MS system can have comparable quantitative performance with QqQ-MRM carried out using a triple quandrupole MS system. Using a mixture of endogenous molecules, we evaluated key analytical merits in quantitative analysis including selectivity, sensitivity/dynamic range, matrix effects, accuracy and precision. Subsequently we used both EISA-MRM and QqQ-MRM methods performed with the same triple quadrupole MS system to calculate the concentrations of investigated metabolites in a plasma extract, a bacterial cell extract, and a mammalian cell extract and the results acquired using both methods were compared. To confirm the applicability of EISA-MRM, we further performed the analysis of a NIST certified plasma sample using EISA-MRM with a single quadrupole MS system.
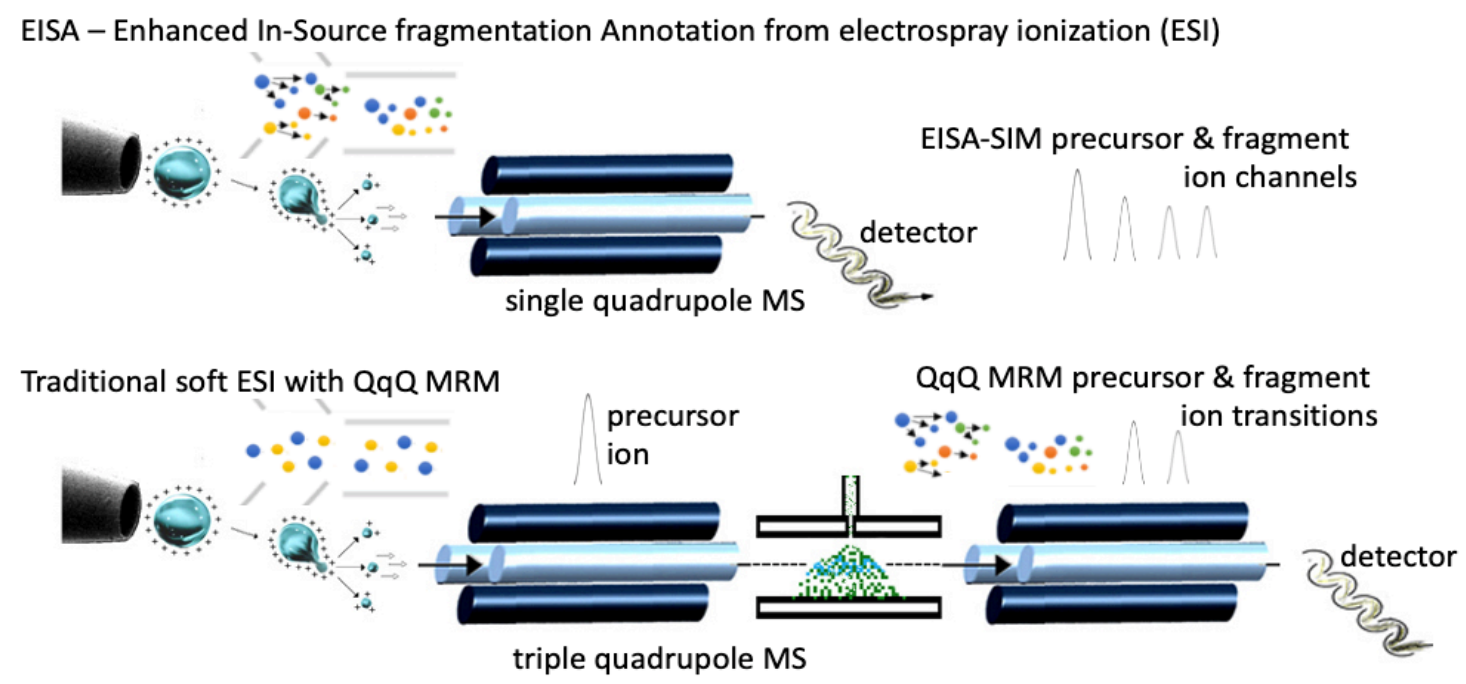

Figure 1. General experimental design for EISA-MRM with single quadrupole mass spectrometry and the more traditional QqQ-MRM triple quadrupole MS system. 


\section{EXPERIMENTAL SECTION}

Materials. A total of 50 endogenous molecules were selected to represent a broad range of physicochemical properties and chemical structures, including amino acids, lipids, and fatty acids. For the investigation of quantitative performance of EISA-MRM including the limit of quantification (LOQ) and dynamic range, the fifty molecules were prepared at 9 concentrations spanning 7 orders of magnitude: $0.2 \mathrm{nM}, 0.5 \mathrm{nM}, 1 \mathrm{nM}, 10 \mathrm{nM}, 100 \mathrm{nM}, 1 \mu \mathrm{M}, 10 \mu \mathrm{M}, 100 \mu \mathrm{M}$, and $1 \mathrm{mM}$. In assessing the linearity of calibration line, additional calibration standards were used when necessary (calibration points within the linearity ranges were less than five). For investigating matrix effects in EISA-MRM analysis, plasma (both human male AB plasma and NIST SRM 1950 plasma), mixtures of two mammalian cell lines (VERO C1008 and L6 myocyte), and bacteria cells (Pantoea strain sp. MT058) isolated from the groundwater in Oak Ridge Reservation were used. Standards and both plasma samples were purchased from SigmaAldrich (St. Louis, MO). VERO C1008 cell line and L6 myocyte cell line were obtained from James E. Voss laboratory in the Scripps Research Institute (La Jolla, CA) and Olivia Osborn laboratory in the University of California San Diego, respectively. Bacteria cell pellets were shipped from Michael W. Adams laboratory at University of Georgia and Nitin S. Baliga laboratory in the Institute for Systems Biology.

Sample preparation. Plasma samples were prepared using protein precipitation for both matrix preparation and amino acids extraction ${ }^{17}$. Briefly, $400 \mu \mathrm{L}$ solvent (acetonitrile:methanol; 1:1) was added to $100 \mu \mathrm{L}$ plasma sample. After storage at $-20^{\circ} \mathrm{C}$ for 1 hour, the sample was sonicated on ice for $10 \mathrm{~min}$, followed by centrifugation at $13,000 \times \mathrm{g}$ at $4{ }^{\circ} \mathrm{C}$ for $15 \mathrm{~min}$. The supernatant was collected and dried in a vacuum concentrator (LABCONCO) at $10^{\circ} \mathrm{C}$ and reconstituted with $100 \mu \mathrm{L}$ acetonitrile:water (1:1). After sonication (10 min, on ice) and centrifugation $\left(13,000 \times \mathrm{g}, 4^{\circ} \mathrm{C}, 15 \mathrm{~min}\right)$, the supernatant was transferred to a LC-MS glass vial with inserts for instrumental injection and for preparing matrix matched calibration lines. For the extraction of total fatty acids from plasma sample, a mixture of hexane/isopropanol (3:2) was used and $0.3 \mathrm{M} \mathrm{KOH}$ in $80 \%$ methanol was used for alkaline hydrolysis, with details documented elsewhere ${ }^{18}$.

Bacterial and mammalian cells were extracted using a solvent mixture of acetonitrile:methanol:water $(2: 2: 1)^{19}$. In brief, cell samples ( 1 million cells in each tube) were sonicated on ice for 15 min after shock-freezing in liquid nitrogen and subsequent thawing at 
room temperature. The operation was repeated for three times. Then the sample was incubated at $-20^{\circ} \mathrm{C}$ for one hour allowing protein precipitation, followed by centrifugation at $13,000 \times \mathrm{g}$ at 4 ${ }^{\circ} \mathrm{C}$ for $15 \mathrm{~min}$. Using the same procedure as mentioned above, the supernatant was collected, dried and reconstituted with $50 \mu \mathrm{L}$ acetonitrile:water (1:1). After sonication (10 min, on ice) and centrifugation $\left(13,000 \times \mathrm{g}, 4^{\circ} \mathrm{C}, 15 \mathrm{~min}\right)$, the supernatant was transferred to a LC-MS glass vial with inserts for instrumental injection and for preparing matrix matched calibration lines.

Triple quadrupole mass spectrometry analysis. EISA-MRM and QqQ-MRM experimental methods were carried out on a Waters Xevo TQ-XS triple quadrupole mass spectrometry system (Milford, MA) to allow for consistent comparison without the variability associated with using different instruments. Collision gas flow on the triple quadrupole was turned off when running the EISA-MRM experiment and the first quadruple was used for analysis. The analyses were performed in both positive and negative electrospray ionization modes, and the EISA-MRM and QqQ-MRM transitions measured for each molecule are shown in Table S1. In EISA-MRM method, cone voltage was optimized for each molecular ion (precursor ion and fragments) and the optimal cone voltage with the highest intensity was used. In QqQ-MRM method, optimal cone voltage of the precursor ion was used for every corresponding transition, and optimal collision energy was acquired for each fragment ion. A dwell time of $15 \mathrm{~ms}$ was used for each channel in EISA-MRM method and each transition in QqQ-MRM method.

ACQUITY UPLC BEH C18 column $(2.1 \times 100 \mathrm{~mm}, 1.7 \mu \mathrm{m}$, Waters $)$ and ACQUITY UPLC BEH Amide column $(2.1 \times 100 \mathrm{~mm}, 1.7 \mu \mathrm{m}$, Waters $)$ were used in the separation of metabolites in reverse phase and HILIC analysis, respectively. For the reverse phase analysis, metabolites were separated by gradient elution at a flow rate of $200 \mu \mathrm{L} / \mathrm{min}$ starting at $25 \%$ (v/v) $\mathrm{B}$, held for $1 \mathrm{~min}$, increased to $99 \% \mathrm{~B}$ within $8 \mathrm{~min}$, held for $3 \mathrm{~min}$, and reverted to $25 \% \mathrm{~B}$ at 12.1th $\mathrm{min}$, held for $2.9 \mathrm{~min}$, with a total run time of $15 \mathrm{~min}$. The mobile phases comprised acetonitrile:water (60:40) containing $0.1 \%$ formic acid and $1 \mathrm{mM}$ ammonium formate (A) and isopropanol:acetonitrile (90:10) containing 0.1\% formic acid and $1 \mathrm{mM}$ ammonium formate (B). For the HILIC analysis, metabolites were separated by gradient elution at a flow rate of 250 $\mu \mathrm{L} / \mathrm{min}$ starting at $5 \%(\mathrm{v} / \mathrm{v}) \mathrm{A}$, increased to $80 \%$ A within $8 \mathrm{~min}$, held for $0.5 \mathrm{~min}$, and reverted to $5 \% \mathrm{~A}$ at $8.6^{\text {th }} \mathrm{min}$, held for $3.9 \mathrm{~min}$, with a total run time of $12.5 \mathrm{~min}$. The mobile phases comprised water containing $10 \mathrm{mM}$ ammonium acetate and $0.1 \%$ formic acid (A) and water/acetonitrile (5:95) containing $0.1 \%$ formic acid (B). 
Single quadrupole mass spectrometry analysis. EISA-MRM analysis of 11 amino acids (positive mode) and 5 fatty acids (negative mode) as well as their respective isotope labelled standards was accomplished on an Agilent 6130 Quadrupole LC-MS system (Santa Clara, CA). The ESI source parameters in both positive and negative modes were set as follows: drying gas flow $9 \mathrm{~L} / \mathrm{min}$, drying gas temperature $350{ }^{\circ} \mathrm{C}$, and nebulizer pressure $30 \mathrm{psig}$. Capillary voltage was set at $4000 \mathrm{~V}$ in positive mode and $3500 \mathrm{~V}$ in negative mode, respectively. Fragmentor voltage was optimized for each monitored ion and dwell time was $290 \mathrm{~ms}$ at each channel.

ACQUITY UPLC BEH C18 column $(2.1 \times 100 \mathrm{~mm}, 1.7 \mu \mathrm{m}$, Waters $)$ was used in the analysis of fatty acids, and ACQUITY UPLC BEH Amide column $(2.1 \times 100 \mathrm{~mm}, 1.7 \mu \mathrm{m}$, Waters) was used in the analysis of amino acids. The gradient in fatty acid analysis was as follows: starting at $20 \%(\mathrm{v} / \mathrm{v}) \mathrm{B}$, held for 1 min, increased to $95 \%$ B within 12 min, held for 1 min, and reverted to $20 \% \mathrm{~B}$ at $14.5 \mathrm{~min}$, held for $3.5 \mathrm{~min}$, with a total run time of $18 \mathrm{~min}$. The gradient in amino acid analysis was as follows: starting at 10\% (v/v) A, held for 2 min, increased to $70 \% \mathrm{~A}$ within $11 \mathrm{~min}$, and reverted to $10 \% \mathrm{~A}$ at $11.5 \mathrm{~min}$, held for $3.5 \mathrm{~min}$, with a total run time of $15 \mathrm{~min}$. The mobile phases used in fatty acid and amino acid analysis were the same as those used in reverse phase and HILIC analysis in the triple quadrupole mass spectrometry analysis, respectively. The mobile phase flow rate was set as $100 \mu \mathrm{L} / \mathrm{min}$ in the analysis of both fatty acids and amino acids.

Data analysis. Extracted ion chromatograms (EICs) generated using both EISA-MRM and QqQMRM methods were manually inspected with vendor specific software. Target peak was carefully selected based on both precursor/fragment ions and retention time. The peak intensity and area were recorded for either comparison or quantification purpose. One molecule typically has four EIC peaks (one precursor ion and three fragments) through EISA-MRM method, and three EIC peaks (three MRM transitions corresponding to the three fragments) through QqQMRM method. Selection of the quantifier was based on the linear dynamic range of each molecular ion or transition and the concentration of target metabolites in the test sample.

Molecular standards of all 50 molecules at $10 \mu \mathrm{M}$ were injected using both EISA-MRM and QqQ-MRM methods in the same triple quadrupole MS system to compare peak intensity of the same fragment ion. After that, limits of quantification (LOQs) and linear dynamic ranges (LDRs) of the 50 molecules were investigated for each acquisition method using a total of 9 concentration levels in solvent. LOQ was defined as the lowest analyte concentration that can be 
quantitatively detected with acceptable accuracy and precision (back calculated accuracy within $30 \%)$. Using the solvent based calibration lines $\left(1 / \mathrm{x}^{2}\right.$ weighted) acquired through both methods, we calculated the concentrations of the fifty metabolites in a bacteria cell extract; for metabolites not endogenously present in the bacteria cell extract, we spiked the analytical standards into the sample at varying concentrations ranging from $10 \mathrm{nM}$ to $100 \mu \mathrm{M}$ to increase the metabolite coverage for quantitative performance evaluation. We further calculated the concentrations of these metabolites in a plasma extract and a mammalian cell extract mixture to investigate the performance of EISA-MRM in different types of matrices. Every test sample was injected for five times and the averaged results were used. Coefficient of variation (CV) was used to assess the precision of replicate analysis.

The biological matrices mentioned above, including plasma, bacterial cell extract, and mammalian cell extract, were selected as the typical biological matrix to investigate the matrix effect of EISA-MRM. Based on the results measured, the 50 metabolites were classified into two groups: those endogenously present in the sample and those not detected. For those metabolites endogenously present, standard addition method was used to investigate the matrix effects at seven concentration levels, namely $0.1,1,5,10,50,100$, and $200 \mu \mathrm{M}$. Then matrix effect was assessed by comparing the peak area of the analyte standard in solvent and matrix at the same concentration, defined as [(peak area in solvent/in matrix $) \times 100]$. For those metabolites not present or at low levels $(<1 \mathrm{nM})$ in the target matrix, matrix matched calibration lines were prepared at concentrations ranging from $10 \mathrm{nM}$ to $200 \mu \mathrm{M}$ by spiking standards into the postextraction samples, referred as analyte free matrix method. Here, slopes of the linear calibration lines for the same set of standards generated in both solvent and matrix were compared to assess the matrix effect. Each standard was injected for five times and the averaged peak area or slope was used in the analysis.

The accuracy and precision of EISA-MRM in quantitative analysis was further evaluated by measuring the amino acids and fatty acids in a certified NIST SRM 1950 plasma sample using isotope dilution-based plasma matrix matched calibration curve. The intra- and inter-day precision of the method was determined by replicate analyses. The linearity of each calibration line was confirmed by plotting the peak area ratio of metabolite standard to its isotope labelled standard versus metabolite concentration. The sample concentrations were calculated from the equation $y=a x+b$, as determined by weighted $\left(1 / x^{2}\right)$ linear regression of the calibration line. The 
accuracy of the method was expressed as [(mean observed concentration)/(certified/reference mean concentration) $] \times 100$.

\section{RESULTS AND DISCUSSION}

\section{In-Source Fragments Generation in EISA-MRM}

In earlier work, we demonstrated that enhanced in-source fragmentation allowed for the production of both high abundant precursor ions and fragment ions that were characteristic of tandem mass spectrometry data for the majority of molecules investigated, thus enabling untargeted metabolomic experiments with single mass analyzers. In this effort we extend EISA as a quantitative technology platform demonstrating that EISA can also facilitate the quantitative analysis of small molecules in a single quadrupole MS system by producing the high abundant precursor ion and its respective fragments simultaneously. This can significantly improve the applicability of a single quadrupole MS system in quantitative analysis, which typically only uses the precursor ion in selecting the target analyte.

To investigate the in-source fragments generation performance of EISA in EISA-MRM quantitation method, we prepared a group of fifty molecules containing a variety of chemical structures including amino acids, sugars, fatty acids, and lipids (Table S1). Except the fatty acids analyzed in the negative mode, all other molecules produced at least 3 fragments (Table S1). Insource fragmentation was performed with a triple quadruple to enable direct comparison of single and triple quadrupole performance on the same instrumentation. For example, the cone voltage in the Waters triple quadrupole MS system, was optimized for each ion including both precursor and fragment ions to acquire their own maximum peak intensities. With the increase of cone voltage, peak intensity of molecular ions either increase or a relationship of reversed $\mathrm{U}$ shape curve is observed (Fig. S1). In EISA-MRM, the optimal cone voltage of each ion was used in preparing the quantitative analysis method to simultaneously acquire the precursor ion and its fragments at their own peak intensities.

When running the EISA-MRM experiments on the triple quadrupole MS system, collision gas flow was turned off and the first quadruple was used for analysis. In addition, for direct comparison purposes, we used QqQ-MRM to analyze these same collision-induced fragments generated in the collision cell for all the target molecules from the same instrument that the EISA-MRM experiments were performed. Maximum intensity was also acquired for 
each fragment ion by adjusting the relevant parameters like collision energy. Comparison of EISA-MRM and QqQ-MRM chromatograms of identical standards (10 $\mu \mathrm{M}$ each) revealed that $86 \%$ of the fragments acquired using EISA-MRM had higher peak intensity with a median increase of around $300 \%$ and $400 \%$ in electrospray ionization positive and negative mode, respectively (Fig. 2a), with an overall median increase of 260\% in peak intensity (Fig. S2). We investigated the impact of EISA on metabolites within different chemical classes and found that the median increase of fragment ion intensity for lipids and fatty acids were over $800 \%$, while it was over $300 \%$ for other molecules, indicating that EISA provides different enhancements across different chemical classes (Fig. 2b). For example, lipids and fatty acids appear to have enhanced fragmentation in EISA. This analyte dependent fragmentation in EISA can be further developed for optimizing quantitative analysis.

a

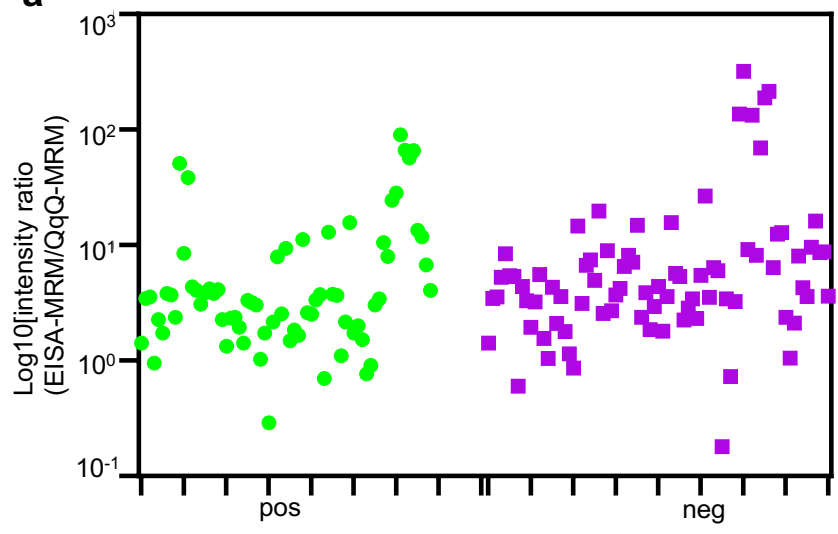

C

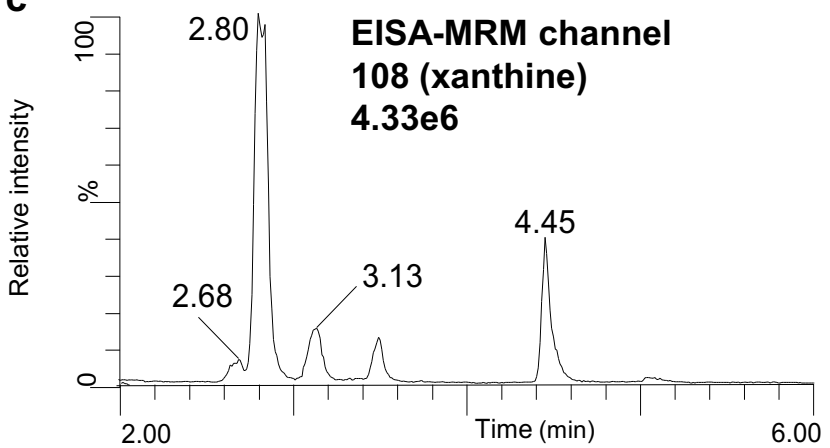

b

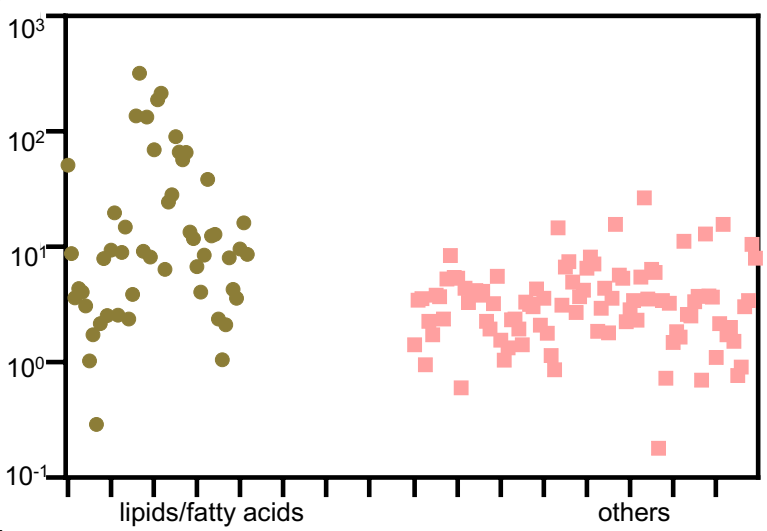

d

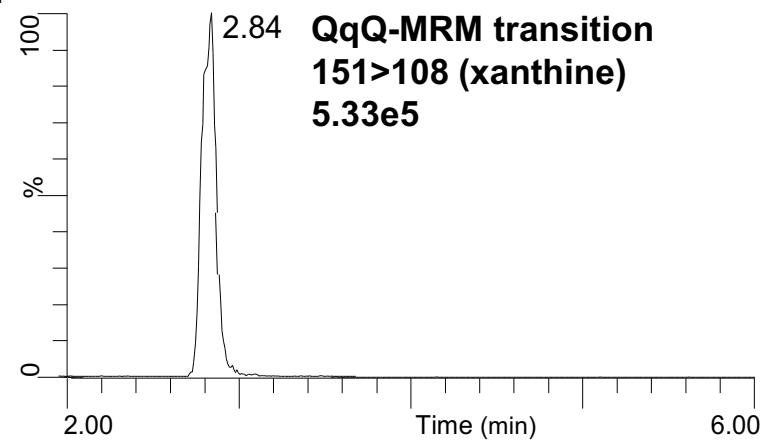

Figure 2. Fragment ion intensity ratio (EISA-MRM/QqQ-MRM) comparison between electrospray ionization positive mode and negative mode (a); Fragment ion intensity ratio (EISA-MRM/QqQMRM) comparison between lipids/fatty acids and other molecules (b); A typical extracted ion chromatogram of an EISA-MRM channel (xanthine in solvent, fragment ion 108 at RT 2.80 min) (c); A typical extracted ion chromatogram of a QqQ-MRM transition (xanthine in solvent, MRM transition 151>108 at RT $2.84 \mathrm{~min}$ ) (d). Log transformed intensity ratio was used in this figure. RT, retention time. The number next to the EISA-MRM channel or QqQ-MRM transition in (c) and (d) represents the maximum intensity of the EIC. 


\section{Selectivity/Specificity}

EISA-MRM would seemingly suffer from limited selectivity when compared to QqQMRM analysis, lacking the ability to generate transitional mass spectrometric pairs (as in QqQMRM) deriving from a selected precursor. However, EISA takes advantage of its ability to simultaneously produce both high abundant precursor and fragment ions originating from the same molecule in a single quadrupole MS system. Therefore, by applying criteria originally adopted for GC/MS based selected ion monitoring quantitation described in EU Commission Directive 2002/657/EC ${ }^{14}$, we were able to generate quantitative data, similar to what has been previously accomplished with GC/MS and QqQ-MRM. While QqQ-MRM exploits a tandem quadrupole mass analyzer's ability to selectively and simultaneously analyze both precursor and fragment ions resulting in four identification points (according to 2002/657/EC Directive), EISAMRM implements this concept by monitoring one precursor ion and three respective fragments (termed "channels"). The resultant EISA-MRM is composed of 4 channels, thereby equaling four identification points equivalent to two QqQ-MRM transitions with one precursor and two fragment ions, facilitating effective quantitative analysis. Xanthine is provided as an example, with a typical EIC from an EISA-MRM channel and a QqQ-MRM transition (using the same fragment ion) are shown in Fig. 2c and 2d, respectively.

High specificity of QqQ-MRM in quantitative analysis is mainly achieved using specific precursor/fragment ion pairs and retention time. Single quadrupole MS analysis utilizes one mass filter and thus it is not possible to directly link fragments with the associated precursor ion. However, we observed that EISA-MRM can significantly improve the selective capability of target analytes from the complex $\mathrm{MS}^{1}$ spectra by simultaneously monitoring the precursor ion and its high abundant characteristic fragments $(n=3)$ eluted at the same retention time. The insource fragments serve as an identity indicator and can also be used for quantification once specificity is confirmed.

METLIN-MRM ${ }^{10}$ and the SMRT dataset ${ }^{20}$ from the METLIN tandem mass spectra database $^{21}$ has also been implemented to facilitate EISA-MRM (Fig. 3) and help deconvolve commonly co-eluted compounds (Fig. 3a). In addition, interfering molecular ions can be detected by comparing the peak intensity/area ratios between the precursor ion and its fragments (p:f1:f2:f3) across standards and samples (Fig. 3b). This takes advantage of the fact that a peak 
intensity/area ratio between precursor ion and fragment ion stemming from one compound stay constant between standard and sample.

Another approach to enhance EISA-MRM selectivity is through application of orthogonal separation technologies where the additional separate "channels" could be used for identification and/or quantification purposes. For example, ion mobility data could serve as an orthogonal set of information unique to EISA in-source fragment ions, as shown to be separated via drift tube based ion mobility (Fig. S3) and thus act as an alternative means for characterizing these ions in accordance with Directive 2002/657/EC, using CCS values. Fig. S3 further shows tandem mass spectrometry data coinciding with the in-source fragments.

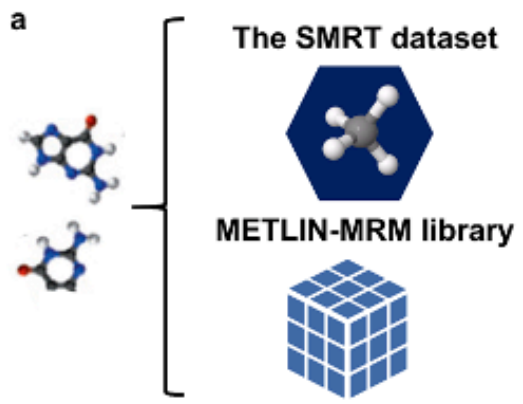

b

$$
\text { Peak intensity/area ratio comparison (p:f1:f2:f3) }
$$
standard

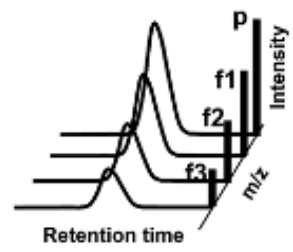

sample

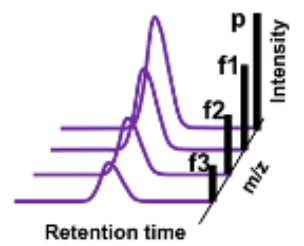

Characteristic fragments of coeluted metabolites

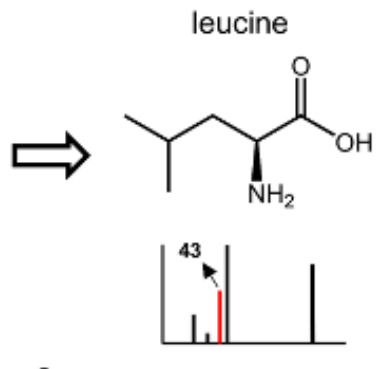

c

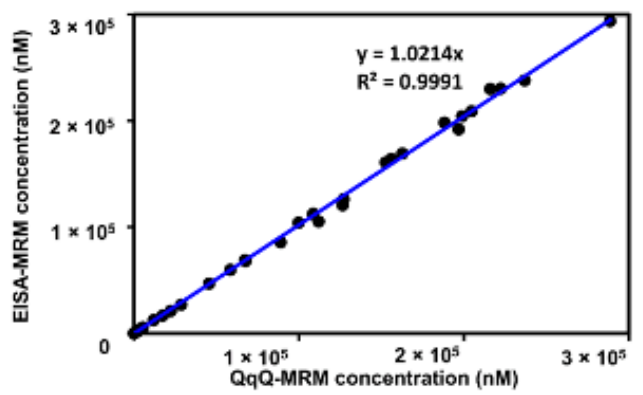

Figure 3. Characteristic fragments of coeluted metabolites can be selcted using the SMRT dataset and METLIN-MRM library with leucine and isoleucine as examples (a). Certain algorithms can be developed to check the specificty of quantifier used in EISA-MRM method, for example, algothrim based on the peak intensity ratio (between precursor ion and fragment ions) comparison between standard and sample (b). The correlation of concentrations of 50 metabolites calculated between EISA-MRM and QqQ-MRM methods in the identical cell sample (analytical standards were spiked into the cell sample at varying concentrations if the metabolites were not detected in the cell sample).

\section{Sensitivity/Dynamic Range}

A wide linear dynamic range (LDR) is important for small molecule quantitation in biological samples. According to the Human Metabolome Database, metabolite concentrations in 
human plasma and urine broadly vary from the pico- up to the millimolar range $\mathrm{e}^{22,23}$. Having a wide LDR makes experimentation simpler by avoiding re-analysis and additional dilutions. The LDR can be limited either by the LOQ or by saturation at the ion source and detector ${ }^{24}$. Thus, we prepared solvent based calibration lines for fifty molecules at nine concentration levels ranging from $0.2 \mathrm{nM}$ to $1 \mathrm{mM}$ to compare the LDRs for the same compound between EISA-MRM and QqQ-MRM ${ }^{15}$. Weighted $\left(1 / \mathrm{x}^{2}\right)$ linear regression calibration lines were established for each molecule by plotting the relationship between concentration level and the corresponding peak area. LOQs were determined as the lowest concentration level in the calibration line with back calculated accuracy values between $70-130 \%$, which were also used as the lower limit of the LDR. The upper limit in the LDR was determined using the same criterion. Here, only those molecules containing 3 fragments were analyzed and several molecules which didn't show linear relationship between the concentration level and peak area were excluded. Finally, LDRs of a total of 43 molecules acquired using both EISA-MRM and QqQ-MRM methods are reported (Table S2). These molecules occupied the majority HILIC and reverse phase space in both positive and negative modes.

We first acquired the LDRs for each molecular ion (EISA-MRM) or MRM transition (QqQ-MRM), then an integrated LDR was reported for each molecule in each method, combing the lower limit of the LDR of a primary sensitive ion and the upper limit of the LDR of a less sensitive ion. It was expected that sensitivity in EISA-MRM is going to be lower compared to QqQ-MRM for the same molecule because of higher background noise levels observed in the EIC when only one mass filter is used. However, our results clearly showed that EISA-MRM can achieve the same sensitivity for most analytes in quantitative analysis. Of the 43 analytes, only around 30\% of molecules had one order of magnitude lower LOQs in EISA-MRM than QqQMRM. Particularly, we observed that five molecules, including glucose, methylhistidine, glutathione, C20 sphingosine, and oleic acid, had LOQs of one order of magnitude higher in EISA-MRM, indicating that certain chemicals can be more efficiently detected in EISA-MRM (Table S2). Thus, although there is a higher chemical noise baseline in the EIC produced by the EISA-MRM method, the majority of chemicals can still maintain the same LOQs as those produced using the QqQ-MRM method. Further, we found that around 90\% of molecules had the same or higher upper limit of the LDR in EISA-MRM. This indicates that saturation points were the same between the two methods for most ion signals and only around $10 \%$ of molecules get 
saturated relatively easier in EISA-MRM. Overall, EISA-MRM can achieve a LDR of up to 5 orders of magnitude, for over $90 \%$ of the analytes investigated.

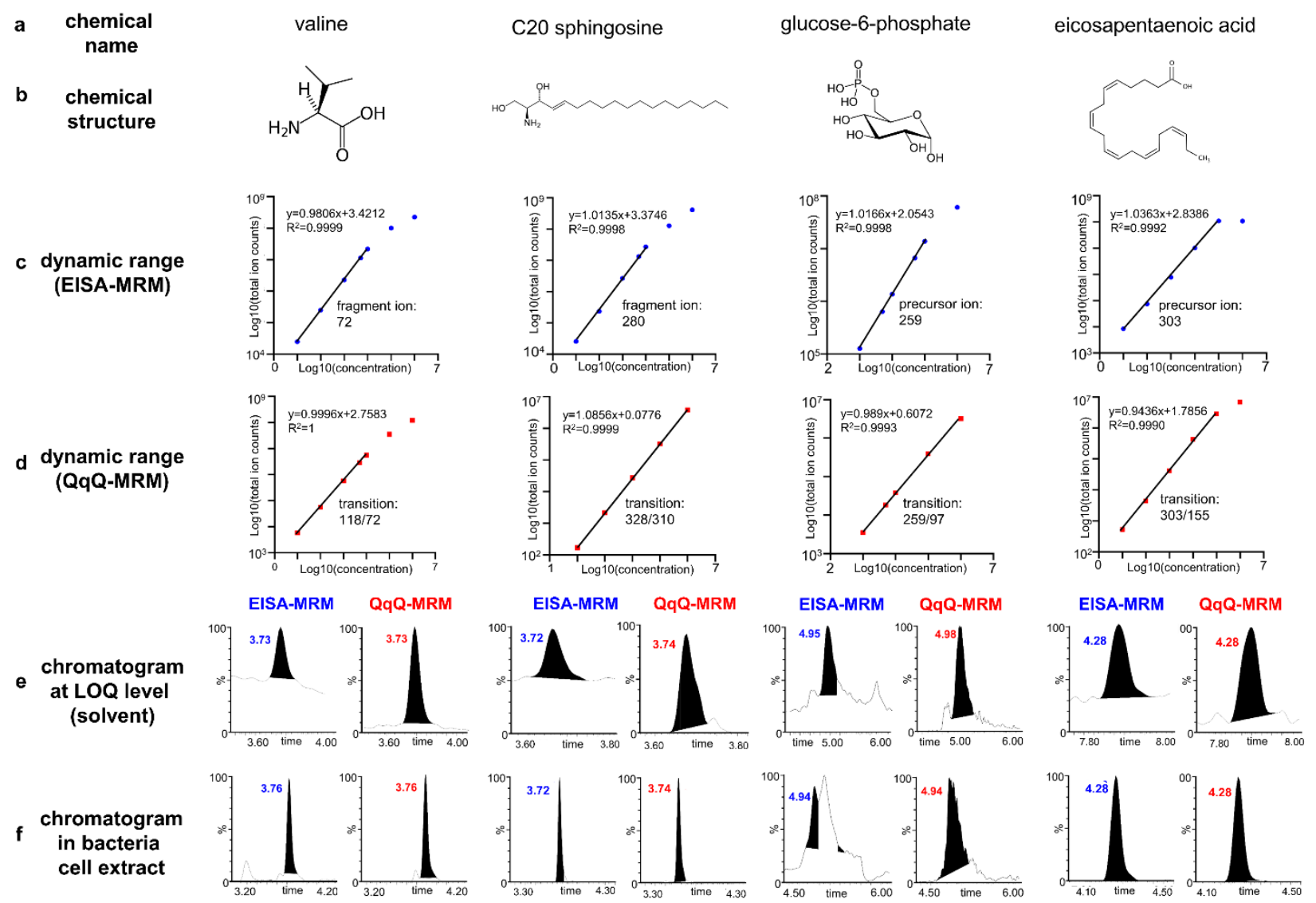

Figure 4. Chemical structures (a, b), dynamic ranges (c, d), LC chromatograms at LOQ levels (e), and LC chromatograms in bacterial cell extracts (f) of four representative molecules with different physicochemical properties. Dynamic ranges and LC chromatograms acquired in both EISA-MRM and QqQ-MRM methods are shown; the linear curves were calculated based on the $\log 10$ transformed values; the molecular ion used in EISA-MRM and the transition used in QqQ-MRM method are shown next to the linear curve. Valine and glucose-6-phosephate were acquired using the HILIC column; C20 sphingosine and eicosapentaenoic acid were acquired using the reverse phase column.

Four molecules with different physicochemical properties, including valine, C20 sphingosine, glucose-6-phosphate, and eicosapentaenoic acid (EPA), were selected to illustrate the differences in LDR between the two techniques (Fig. 4a and 4b). As shown in Fig. 4c and 4d, EISA-MRM achieves similar or lower (C20 sphingosine) LOQs for the select compounds. Chromatograms of the four molecules at LOQ levels in solvent in both the QqQ-MRM and EISA-MRM modes are shown in Fig. 4e. As illustrated, while EISA-MRM comes with a greater level of chemical noise, the higher ion intensity enables comparable sensitivity. Of the four 
molecules, valine and EPA presented an identical dynamic range between the two modes; EISAMRM analysis of C20 sphingosine proved more sensitive when compared to QqQ-MRM allowing to extend the dynamic range towards lower analyte concentrations; for glucose-6phosphate, detector saturation was observed at $1 \mathrm{mM}$ in EISA-MRM mode, while not in QqQMRM mode. Chromatograms of the four molecules acquired using EISA-MRM and QqQ-MRM modes in a bacteria cell extract are shown in Fig. 4f. In summary EISA-MRM performs equally good or even better in terms of sensitivity and dynamic range for the majority of metabolites investigated.

\section{Application and comparison of EISA-MRM and QqQ-MRM to cell extracts and plasma samples}

Next, we used above mentioned calibration lines to quantify these molecules in a bacteria cell extract using both QqQ-MRM and EISA-MRM methods. For those compounds which were not endogenously present, the bacteria cell extract was fortified with defined amounts of metabolite standards for quantification purposes. The concentrations calculated ranged from 1 $\mathrm{nM}$ to over $100 \mu \mathrm{M}$ levels and good alignment was observed between the results calculated using the two techniques (Fig. 3c; Table S3). We further analyzed these metabolites in a plasma extract and a mammalian cell extract using both QqQ-MRM and EISA-MRM techniques, and concentrations of the detected metabolites are shown in Tables S4 and S5, respectively. The percent difference of the results calculated using the two techniques were below $7 \%$ for over $90 \%$ of the molecules measured in every matrix. Highly coeluting peaks from matrix can interfere the accuracy in peak integration and lead to a higher percent difference, e.g., uric acid in plasma.

\section{Matrix Effect}

A key advantage of QqQ-MRM analysis of complex mixtures is the high selectivity achieved by the combination of two mass filters and a collision cell. As EISA-MRM works with a single quadrupole, selectivity is an important analytical feature that requires evaluation. Thus, we investigated three complex biological matrices, including a plasma extract, a mammalian cell extract, and a bacteria cell extract. As mentioned above, we first analyzed the three matrices and measured the concentrations of fifty metabolites in each of them. For those metabolites which 
were present in the matrices, we used the standard addition method to evaluate the matrix effects by spiking five to eight different concentration levels into the extract post-extraction ${ }^{16}$. For metabolites not endogenously present in the matrices, we spiked eight different concentration levels into each of the three different matrices post-extraction. Matrix effects were evaluated according to Matuszewski et al. ${ }^{16}$ and as described below, for all fifty metabolites in both QqQMRM and EISA-SIM modes. Overall, the two techniques showed similar matrix effect trends (suppression or enhancement) for the same metabolite and matrix. There were no significant differences of the matrix effects observed between QqQ-MRM and EISA-MRM. Five molecules, including phenylalanine, hypoxanthine, uridine, inosine, and guanosine, were selected to demonstrate the matrix effects of molecules in the three different matrices assessed using the standard addition method (Tables S6-S8). We established calibration lines for metabolites which were not present in the sample and compared their slopes in solvent and matrix as well as the standard deviations in replicate injections $(n=5)$ with examples shown in Table 1. Overall, our results indicate that the difference of matrix effects between the two analytical modes are compound and matrix dependent and independent of the analytical technique (EISA-MRM versus QqQ-MRM).

Table 1. Slopes of calibration lines for inosine in plasma extract, hypoxanthine in mammalian cell extract, and glucose-6-phosphate in bacteria cell extract as well as their calibration lines in solvent using both QqQ-MRM and EISA-MRM quantification techniques.

\begin{tabular}{|c|c|c|c|c|c|c|}
\hline & \multicolumn{3}{|c|}{ QqQ-MRM } & \multicolumn{3}{|c|}{ EISA-MRM } \\
\hline & solvent & $\begin{array}{c}\text { plasma extract } \\
\text { (inosine) }\end{array}$ & Mann-Whitney test & solvent & $\begin{array}{l}\text { plasma extract } \\
\text { (inosine) }\end{array}$ & $\begin{array}{c}\text { Mann-Whitney } \\
\text { test } \\
\end{array}$ \\
\hline slope $^{a}$ & 195.4 & 197.1 & $p>0.01$ & 2356.2 & 2389.8 & $p>0.01$ \\
\hline$S D^{b}$ & 2.4 & 6.5 & & 46.8 & 109.3 & \\
\hline \multirow[t]{2}{*}{$\mathrm{CV}(\%)^{\mathrm{C}}$} & 1.2 & 3.3 & & 2 & 4.6 & \\
\hline & solvent & $\begin{array}{l}\text { bacteria cell } \\
\text { (glucose-6- } \\
\text { phosphate) }\end{array}$ & Mann-Whitney test & solvent & $\begin{array}{l}\text { bacteria cell } \\
\text { (glucose-6- } \\
\text { phosphate) }\end{array}$ & $\begin{array}{c}\text { Mann-Whitney } \\
\text { test }\end{array}$ \\
\hline slope & 15 & 24.1 & $p<0.01$ & 200.4 & 381.2 & $p<0.01$ \\
\hline SD & 0.6 & 1.2 & & 2.4 & 5.2 & \\
\hline $\mathrm{CV}(\%)$ & 3.9 & 4.8 & & 1.2 & 1.4 & \\
\hline
\end{tabular}

aslope, calculated using the linear calibration line between total ion counts (y) and concentration (x) of the analyte; ${ }^{\mathrm{b}} \mathrm{SD}$, standard deviation between slopes $(n=5) ;{ }^{\circ} \mathrm{CV}$, coefficient of variation, calculated as the percent ratio between the standard deviation and mean value of the slope.

\section{Accuracy and Precision of EISA-MRM Analysis in NIST Certified Plasma Sample}

The analytical accuracy, reproducibility and repeatability are key aspects of any quantitation method. Here, we established quantitative isotope dilution-based analysis method 
using EISA-MRM on a single quadrupole MS system and analyzed 11 amino acids and 5 fatty acids in a certified NIST 1950 plasma to investigate the analytical accuracy and precision of the here described EISA-MRM approach. Calibration lines were prepared using the ratio between external calibrations standards and the corresponding isotopically labelled standards versus the concentrations of target analytes. The results were compared to the certified or reference values provided by NIST for accuracy analysis. The precision was calculated as coefficient of variation (CV) from replicate analysis. CV was calculated as the standard deviation of observed metabolite concentration in the plasma divided by the mean measured concentration. Intra-day precision was calculated on a single day with five replicate injections, while inter-day precision was calculated for five separate days.

As shown in Table 2, the results acquired using EISA-MRM technique in the single quadrupole MS system demonstrated excellent accuracy (91-110\% for amino acids; 76-129\% for fatty acids) and interday precision $(\mathrm{CV}<10 \%)$. The wider accuracy range for fatty acids may be partly ascribed to the different sample analysis protocol and instrument used by NIST (GC-MS). The LC system (Agilent 1260 infinity) coupled with the single quadrupole mass spectrometry system was not able to handle the high pressure, thus leucine and isoleucine were not distinguished here. Therefore, a combined result for the two compounds was reported, which had a relative higher interday $\mathrm{CV}(8.7 \%)$.

Table 2. Concentrations of 11 amino acids and 5 fatty acids in NIST SRM 1950 plasma sample

\begin{tabular}{cccccc}
\hline & \multirow{2}{*}{$\begin{array}{c}\text { certified/reference } \\
\text { concentration } \\
\text { metabolite }\end{array}$} & $(\mu \mathrm{M})$ & intraday & \multicolumn{2}{c}{ interday } \\
\cline { 3 - 6 } & & $\begin{array}{c}\text { accuracy }^{\mathrm{b}} \\
(\%)\end{array}$ & $\begin{array}{c}\mathrm{CV}^{\mathrm{c}} \\
(\%)\end{array}$ & $\begin{array}{c}\text { accuracy }^{\mathrm{b}} \\
(\%)\end{array}$ & $\begin{array}{c}\mathrm{CV}^{\mathrm{c}} \\
(\%)\end{array}$ \\
\hline
\end{tabular}




\begin{tabular}{|c|c|c|c|c|c|c|c|}
\hline & & $\begin{array}{c}\text { measured } \\
\text { concentration } \\
(\mu \mathrm{M})\end{array}$ & & & $\begin{array}{l}\text { neasured } \\
\text { ceentration } \\
(\mu \mathrm{M})\end{array}$ & & \\
\hline histidine & $72.6 \pm 3.6$ & 67.9 & 93.6 & 1.1 & 66.2 & 91.2 & 2.1 \\
\hline leucine/isoleucine & $155.9 \pm 9.7$ & 164.2 & 105.3 & 5.6 & 160 & 102.6 & 8.7 \\
\hline lysine & $140 \pm 14$ & 134.5 & 96.1 & 0.4 & 136.7 & 97.6 & 0.8 \\
\hline methionine & $22.3 \pm 1.8$ & 22.2 & 99.7 & 1.4 & 21.3 & 95.5 & 2 \\
\hline proline & $177 \pm 9$ & 172.3 & 97.3 & 1.1 & 174.7 & 98.7 & 1.4 \\
\hline threonine & $119.5 \pm 6.1$ & 109.3 & 91.4 & 0.2 & 110.1 & 92.1 & 0.4 \\
\hline tyrosine & $57.3 \pm 3.0$ & 54.9 & 95.9 & 1.3 & 54.5 & 95.1 & 2.8 \\
\hline valine & $182.2 \pm 10.4$ & 181.3 & 99.5 & 0.6 & 181.8 & 99.8 & 0.7 \\
\hline phenylalanine & $51 \pm 7$ & 46.5 & 91.2 & 2.8 & 46.8 & 91.8 & 2.5 \\
\hline arginine & $81.4 \pm 2.3$ & 85.8 & 105.4 & 1.1 & 88 & 108.1 & 3.3 \\
\hline $\begin{array}{l}\text { eicosapentaenoic } \\
\text { acid }\end{array}$ & $38.6 \pm 0.5$ & 36.9 & 95.7 & 4.4 & 35.9 & 93 & 4.6 \\
\hline linoleic acid & $2838 \pm 143$ & 2081 & 73.3 & 4.9 & 2157.9 & 76 & 5.3 \\
\hline oleic acid & $1614 \pm 154$ & 1450 & 89.9 & 3.8 & 1456 & 90.2 & 5.3 \\
\hline myristic acid & $80.1 \pm 17.0$ & 98.6 & 123 & 3.6 & 98.3 & 122.7 & 3 \\
\hline stearic acid & $644 \pm 41$ & 822 & 127 & 1.3 & 829.7 & 128.8 & 1.4 \\
\hline
\end{tabular}

\section{CORRELATED SIM CHROMATOGRAM OO CORRELATED ION MONITORING}

EISA-MRM as described above was validated using traditional SIM technology. However, and in order to enhance selectivity and sensitivity, a unique algorithm was created to process EISAMRM data. Unlike SIM, the Correlated SIM Chromatogram (CSC) is designed to correlate and compile multiple ions within one chromatogram. CSC filters for signals only if there is a signal above a certain manually preset threshold in all traces. Therefore, the EISA-MRM chromatogram is created by a compilation of the individual ion signals only if each signal satisfies a particular threshold. In this way individual SIM traces are virtually connected, thereby creating a SIM derived tandem mass spectrometric transition comparable to mass transitions obtained from a QqQ MRM experiment. 
SIM's of Phenylalanine

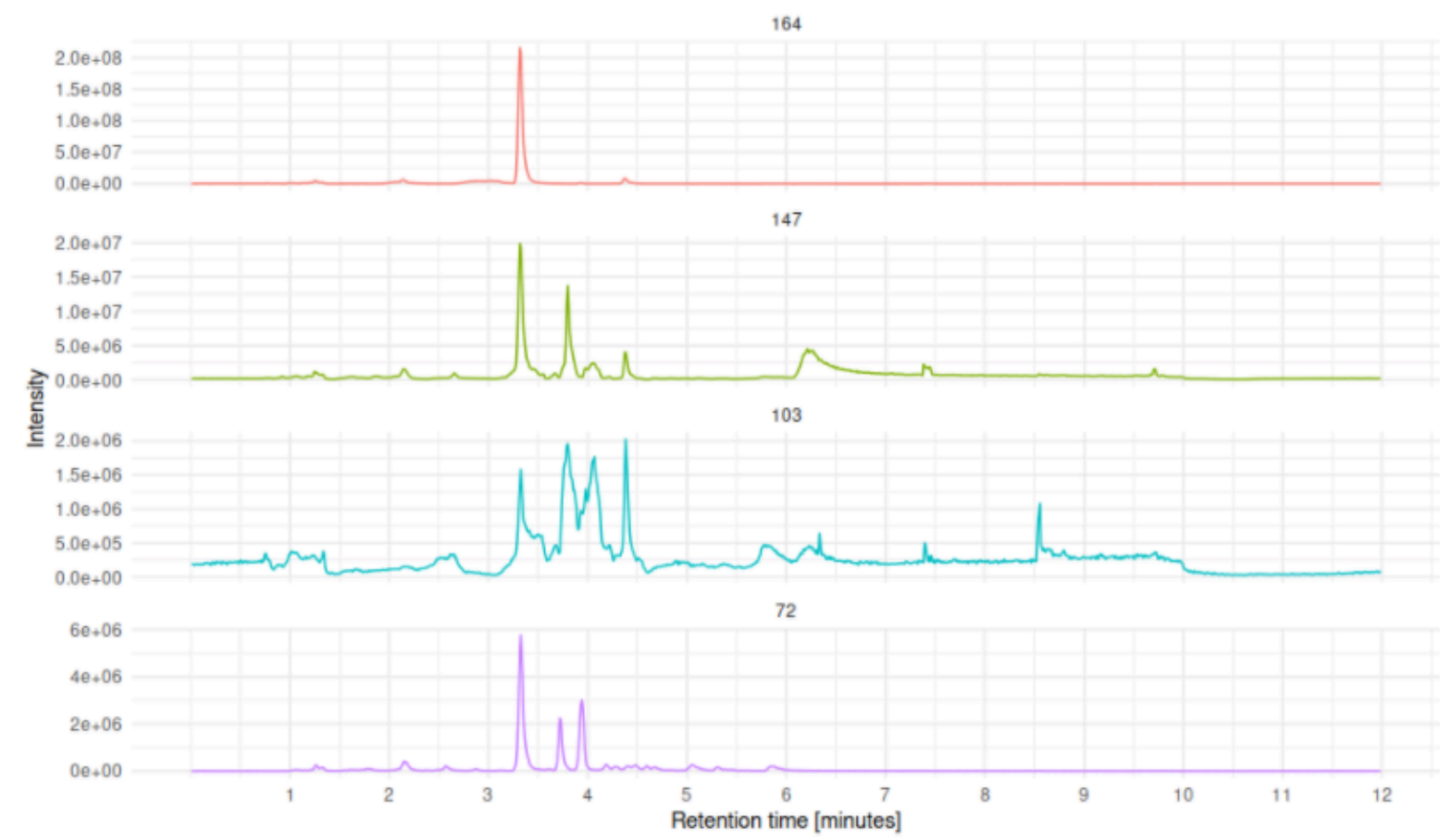

Only keep signal if there is a signal above a certain threshold in all traces. This threshold is set manually. For now the EISA-MRM is create by the precursor signal if there is a signal above the threshold in the precursor signal and the fragment traces the signal of the precursor is kept otherwise the singal will be zero.

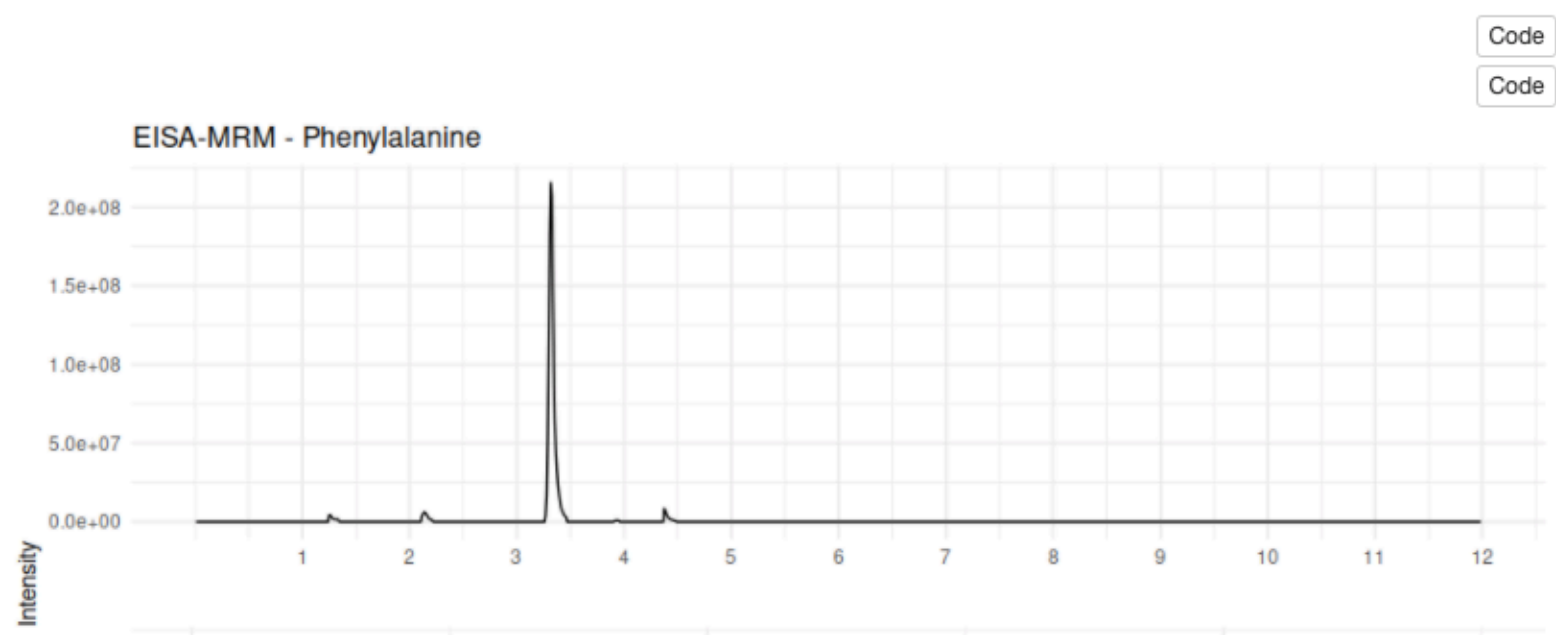

\section{APPLICATIONS AND LIMITATIONS}

Typically, additional MRM transitions have to be performed with a triple quadrupole instrument to enable the identification of quantified compounds, EISA adds this function to the single quadrupole MS system. Thus, EISA-MRM technique can significantly broaden the applications of single quadrupole MS systems in quantitative analysis, especially those used for 
routine analysis. For example, EISA-MRM can be used for reaction monitoring which is performed on a daily basis in synthetic chemistry laboratories ${ }^{25}$. While high resolution and high detection sensitivity are vital features for structure elucidation and trace analysis, reaction monitoring typically requires only unit mass resolution to provide verification of product formation and to track the comings and goings of reaction starting materials, products and intermediate ${ }^{25}$. Further, due to high-maintenance equipment and complicated data analysis, a mass spectrometer (MS) is not widely used in quality control (QC) laboratories in the industry, e.g., pharmaceutical and food industry ${ }^{25}$. However, EISA-MRM can help increase the applications of MS based methods in these QC laboratories, which are normally more sensitive and can substantially reduce the time and cost. For example, EISA-MRM methods can be developed to monitor the post-translational modifications (PTMs) in complex protein drugs ${ }^{26}$. Xu et al. have reported a quadrupole Dalton-based multi-attribute method for PTMs detection and quantitation in a therapeutic monoclonal antibody ${ }^{26}$. This method is shown to be an extremely useful tool for product and process characterization studies that facilitate facile understanding of process impact on multiple quality attributes, while being QC friendly and cost-effective ${ }^{26}$. EISA-MRM methods can also be used in the identification and quantitation of active ingredients, impurities and degradation products in the development of chemical products such as pharmaceuticals and agrochemcials ${ }^{25}$. Besides, EISA-MRM technique can also find applications in a research laboratory in the analysis of biomolecules and xenobiotics ${ }^{25}$.

However, in EISA-MRM analysis, highly co-eluted analytes with precursors and fragment ion masses similar to that of the target compound can result in 'contaminated' transitions, which can lead to false positive identifications or imprecision quantification. Thus, characteristic molecular ions need to be found for the co-eluted target analytes using either tandem mass spectra and retention time databases like METLIN or comparing the signal intensity (peak area) ratios across standards and samples as discussed earlier. Further, QC samples can be added to the sample sequence by adding certain amount of analyte standard to the test sample. By comparing the peak intensity (area) ratio between QC and test samples, molecular ions specific to the target analytes can be determined. If these QC protocols don't work, an extended liquid chromatography gradient is needed to help EISA-MRM improve selectivity, especially for those closely eluted compounds with fragments or molecular ions sharing the same nominal masses. 


\section{CONCLUSIONS}

Conceptually, EISA-MRM alters the traditional tandem mass spectrometry fragmentation and quantitative analysis strategy (as illustrated in Fig. 1a), by instead using a single quadrupole for the analysis of precursor and in-source generated fragment ions. This straightforward utilization of an existing platform facilitates high dynamic range, selectivity, accuracy, broad availability and reproducibility, yet it accomplishes this with a technically simpler platform. In assistance with METLIN, EISA-MRM can be deployed on complex matrices ${ }^{10}$ and also multiple mass spectrometer types (e.g. single quadrupole, triple quadrupole, QTOF, and Q-Orbitrap). This concept is especially intriguing in that the same mass spectrometry system (QTOF and QOrbitrap) can be used, without altering conditions, to perform both full scan and MRM quantitative analyses. Moreover, EISA-MRM can be coupled with other separation technologies including ultra-high resolution capillary electrophoresis and gas chromatography to further enhance their sensitivity. Ion mobility separation could also provide an additional channel for EISA-MRM selectivity. Additionally, using EISA-MRM on QqQ machines would facilitate the development of pseudo- $\mathrm{MS}^{3}$ approaches without the need for linear ion trap technology. This could be particularly useful for the quantification of modified complex molecules such as for example oxidized phospholipids ${ }^{27}$ or closely related eicosanoids ${ }^{28}$.

Taken together, selected ion monitoring has been widely used for the detection and quantitative analysis of small molecules using GC/MS with electron ionization ${ }^{13}$ with single quadrupole mass analysis, EISA-MRM offers an additional function of performing quantitative analyses for a broader range of molecules on the approximately 100,000 electrospray single quadrupole instruments in existence; instruments that are generally inexpensive, easy to operate, and technically less complex.

\section{REFERENCES}

1. Yost, R. A. \& Enke, C. G. Triple quadrupole mass spectrometry for direct mixture analysis and structure elucidation. Anal. Chem. 51, 1251-1264 (1979).

2. Yost, R.A. \& Enke, C.G. Selected ion fragmentation with a tandem quadrupole mass spectrometer. J. Am. Chem. Soc. 100, 2274-2275 (1978).

3. Fenn, J. B. et al. Electrospray ionization for mass spectrometry of large biomolecules. Science 246, 64-71 (1989). 
4. Yuan, M., Breitkopf, S. B., Yang, X., \& Asara, J. M. A positive/negative ionswitching, targeted mass spectrometry-based metabolomics platform for bodily fluids, cells, and fresh and fixed tissue. Nat. Protoc. 7, 872-881 (2012).

5. Addona, T. A. et al. Multi-site assessment of the precision and reproducibility of multiple reaction monitoring-based measurements of proteins in plasma. Nat. Biotechnol. 27, 633-641 (2009).

6. Wang, M. et al. Mass spectrometry searches using MASST. Nat. Biotechnol. 38, 2326 (2020).

7. Venable, J. et al. Automated approach for quantitative analysis of complex peptide mixtures from tandem mass spectra. Nat. Methods 1, 39-45 (2004).

8. Glish, G. \& Vachet, R. The basics of mass spectrometry in the twenty-first century. Nat. Rev. Drug Disco. 2, 140-150 (2003).

9. Mueller, C.A. et al. Development of a multi-target screening analysis for 301 drugs using a QTrap liquid chromatography/tandem mass spectrometry system and automated library searching. Rapid Commun. Mass Spectrom. 19, 1332-1338 (2005).

10. Domingo-Almenara, X. et al. XCMS-MRM and METLIN-MRM: a cloud library and public resource for targeted analysis of small molecules. Nat. Methods 15, 681-684 (2018).

11. Domingo-Almenara, $X$. et al. Autonomous METLIN-guided in-source fragment annotation for untargeted metabolomics. Anal. Chem. 91, 3246-3253 (2019).

12. Xue, J. et al. Enhanced in-source fragmentation annotation enables novel data independent acquisition and autonomous METLIN molecular identification. Anal. Chem. 92, 6051-6059 (2020).

13. Müller, C., Junker, J., Bracher, F., \& Giera, M. A gas chromatography-mass spectrometry-based whole-cell screening assay for target identification in distal cholesterol biosynthesis. Nat. Protoc. 14, 2546-2570 (2019).

14. Commission Decision (2002/657/EC) of 12 August 2002. Implementing Council Directive (96/23/EC) concerning the performance of analytical methods and the interpretation of results. Off J Eur Communities 2002, L221: 8-36.

15. United States Food and Drug Administration (2018). Bioanalytical method validation guide for industry. https://www.fda.gov/media/70858/download (accessed on August 2020)

16. Matuszewski, B. K., Constanzer, M. L., \& Chavez-Eng, C. M. Strategies for the assessment of matrix effect in quantitative bioanalytical methods based on HPLCMS/MS. Anal. Chem. 75, 3019-3030 (2003).

17. Ivanisevic, J. et al. Arteriovenous Blood Metabolomics: A Readout of Intra-Tissue Metabostasis. Sci. Rep. 5, 12757 (2015).

18. Serafim, V. et al. Development and Validation of a LC-MS/MS-Based Assay for Quantification of Free and Total Omega 3 and 6 Fatty Acids from Human Plasma. Molecules 24, 360-361 (2019).

19. Beyer, B. et al. Metabolomics-based discovery of a metabolite that enhances oligodendrocyte maturation. Nat. Chem. Biol. 14, 22-28 (2018).

20. Domingo, $X$. et al. The METLIN small molecule dataset for machine learning-based retention time prediction. Nat. Commun. 10, $5811-5819$ (2019).

21. Xue, J. et al. METLIN $\mathrm{MS}^{2}$ molecular standards database: a broad chemical and biological resource. Nat. Methods 17, 953-954 (2020). 
22. Wishart, D. S. et al. HMDB 3.0-The Human Metabolome Database in 2013. Nucleic Acids Res. 41 (Database issue), D801- D807 (2013).

23. Lisec J., Hoffmann F., Schmitt C. \& Jaeger C. Extending the Dynamic Range in Metabolomics Experiments by Automatic Correction of Peaks Exceeding the Detection Limit. Anal. Chem. 88, 7487-7492 (2016).

24. Liu, H., Lam, L. \& Dasgupta, P.K. Expanding the linear dynamic range for multiple reaction monitoring in quantitative liquid chromatography-tandem mass spectrometry utilizing natural isotopologue transitions. Talanta 87, 307-310 (2011).

25. Bu, X., Regalado, E.L., Hamilton, S.E. \& Welch, C.J. The emergence of low-cost compact mass spectrometry detectors for chromatographic analysis. Trends Anal. Chem. 82, 22-34 (2016).

26. $\mathrm{Xu}, \mathrm{W}$. et al. A Quadrupole Dalton-based multi-attribute method for product characterization, process development, and quality control of therapeutic proteins. MAbs. 9, 1186-1196 (2017).

27. Jónasdóttir, H.S. et al. Detection and structural elucidation of esterified oxylipids in human synovial fluid by electrospray ionization-fourier transform ion-cyclotron mass spectrometry and liquid chromatography-ion trap-MS(3): detection of esterified hydroxylated docosapentaenoic acid containing phospholipids. Anal. Chem. 85, 60036010 (2013).

28. Chakraborty, T. et al. Eicosanoid biosynthesis influences the virulence of Candida parapsilosis. Virulence. 9, 1019-1035 (2018). 